\title{
A NOTE ON EQUIVALENCE CLASSES OF PLATS
}

\author{
By SHINJi FuKuHARA
}

\section{Introduction}

This note is summary of the author's paper [7]. By connecting strings in pairs on the top and the bottom of a braid (Figure 1), we obtain a plat (Figure 2).

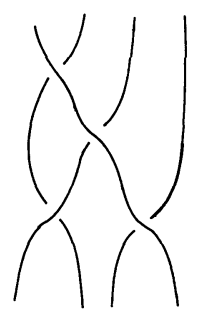

Figure 1

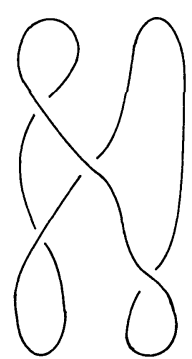

Figure 2

If it comes from a braid with 2 n-strands, it is called a 2n-plat. Two plats are said to be equivalent if there is a homeomorphism $h$ which carries upper and lower halves of $\mathbf{R}^{3}$ to themselves and a plat to the other plat (Figure 3 ).

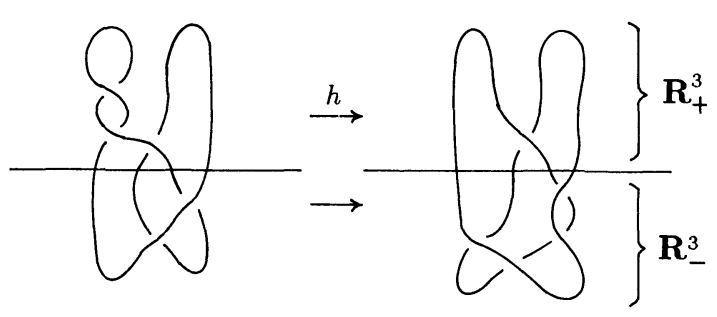

Figure 3

One of the main problems on plats is to decide when two plats are equivalent. In this note we assign a matrix to a plat and show that if two plats are equivalent then assigned matrices are equivalent in our sense. Furthermore we produce a numerical invariant from the matrices. Generally it needs a large amount of calculation to obtain the invariant. But it is quite elementary and can be done by an electric computer. 


\section{Definitions}

Let $\mathbf{R}_{+}^{3}=\left\{(x, y, z) \in \mathbf{R}^{3} \mid z \geqq 0\right\}, \mathbf{R}_{-}^{3}=\left\{(x, y, z) \in \mathbf{R}^{3} \mid z \leqq 0\right\}$ and $\rho: \mathbf{R}_{+}^{3} \rightarrow \mathbf{R}_{-}^{3}$ be defined by $\rho(x, y, z)=(x, y,-z)$. Let $A=A_{1} \cup A_{2} \cup \ldots \cup A_{n}$ be a union of arcs which are properly embedded in $\mathbf{R}_{+}^{3}$ as shown in Figure 4.

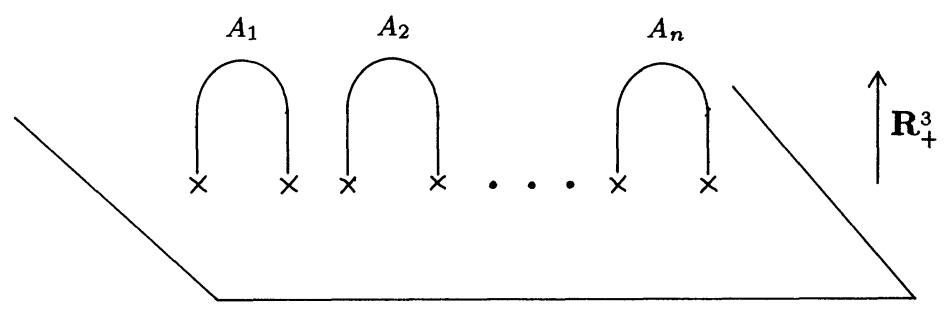

Figure 4

It is assumed that the arcs are unlinked and unknotted. Let $A^{\prime}=A_{1}^{\prime} \cup A_{2}^{\prime} \cup \ldots A_{n}^{\prime}$ be defined by $A_{\imath}^{\prime}=\rho\left(A_{\imath}\right)$ for any $i=1, \ldots, n$. Then $A^{\prime}$ is the union of the $\operatorname{arcs}$ in $\mathbf{R}_{-}^{3}$.

Definition 1. Let $B_{2 n}$ be the braid group of $2 n$-strands. Here in this note we regard an element of $B_{2 n}$ as an isotopy class of an orientation preserving homeomorphism $\varphi:\left(\partial \mathbf{R}_{+}^{3}, \partial A\right) \rightarrow\left(\partial \mathbf{R}_{+}^{3}, \partial A\right)$.

Definition 2. Let $\varphi:\left(\partial \mathbf{R}_{+}^{3}, \partial A\right) \rightarrow\left(\partial \mathbf{R}_{+}^{3}, \partial A\right)=\left(\partial \mathbf{R}_{-}^{3}, \partial A\right)$ be a homeomorphism. The identification space $\left(\mathbf{R}_{+}^{3}, A\right) \cup_{\varphi}\left(\mathbf{R}_{-}^{3}, A^{\prime}\right)$ can be identified with $\mathbf{R}^{3}$ containing $L=A \cup_{\varphi} A^{\prime}$ as a link. In this situation we call $\left(\mathbf{R}_{+}^{3}, A\right) \cup_{\varphi}\left(\mathbf{R}_{-}^{3}, A^{\prime}\right)$ a plat representation of the link $L$. We also call an isotopy class $[\varphi]$ a plat(or $2 n$-plat). Hence we call an element of $B_{2 n}$ a plat in this note.

Next we give definition of equivalence of plats. Two definitions will be considered. One is required to be orientation preserving while the other is not.

Definition 3. Two plats $[\varphi]$ and $[\psi]$ are said (orientation preservingly) equivalent if there is an orientation preserving homeomorphism $h: \mathbf{R}_{+}^{3} \cup_{\varphi} \mathbf{R}_{-}^{\mathbf{3}} \rightarrow \mathbf{R}_{+}^{\mathbf{3}} \cup_{\psi} \mathbf{R}_{-}^{\mathbf{3}}$ which satisfies $h\left(\mathbf{R}_{ \pm}^{3}\right)=\mathbf{R}_{ \pm}^{3}, h(A)=A$ and $h\left(A^{\prime}\right)=A^{\prime}$. In particular if $h$ preserves orientations of $A$ and $A^{\prime}$ it is said to be orientation preservingly equivalent.

Next we define a subgroup $K_{2 n}$ of $B_{2 n}$.

Definition 4. An element $[\varphi]$ of $B_{2 n}$ belongs to $K_{2 n}$ if and only if there is a homeomorphism $\hat{\varphi}:\left(\mathbf{R}_{+}^{3}, A\right) \rightarrow\left(\mathbf{R}_{+}^{3}, A\right)$ such that $\hat{\varphi} \mid \partial \mathbf{R}_{+}^{3}=\varphi$.

Under the definitions we obtain the following lemma.

LEMMA 1. Two plats $[\varphi],[\psi] \in B_{2 n}$ are equivalent if and only if there are $g_{1}, g_{2} \in$ $K_{2 n}$ which satısfy $\varphi=g_{2} \circ \psi \circ g_{1}$. 


\section{Jacobian matrices}

Any plat $\varphi:\left(\partial \mathbf{R}_{+}^{3}, \partial A\right) \rightarrow\left(\partial \mathbf{R}_{+}^{3}, \partial A\right)$ induces an isomorphism $\varphi_{*}: \pi_{1}\left(\partial \mathbf{R}_{+}^{3}-\partial A\right) \rightarrow$ $\pi_{1}\left(\partial \mathbf{R}_{+}^{3}-\partial A\right)$. The group $\pi_{1}\left(\partial \mathbf{R}_{+}^{3}-\partial A\right)$ is identified with a free group of rank $2 n$ by taking $a_{1}, \ldots, a_{n}, b_{1}, \ldots, b_{n}$ in Figure 5 as a generating system.

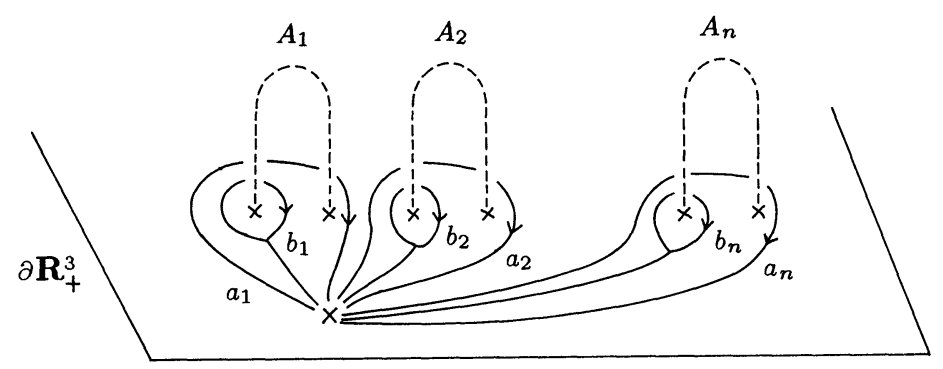

Figure 5

Then $\varphi:\left(\partial \mathbf{R}_{+}^{3}, \partial A\right) \rightarrow\left(\partial \mathbf{R}_{+}^{3}, \partial A\right)$ induces an isomorphism $\varphi_{*}: F\left(a_{1}, \ldots, a_{n}\right.$, $\left.b_{1}, \ldots, b_{n}\right) \rightarrow F\left(a_{1}, \ldots, a_{n}, b_{1}, \ldots, b_{n}\right)$. Let $G$ denote an infinite cyclic group $\mathbf{Z}$ when we work in oriented case and a cyclic group $\mathbf{Z}_{2}$ of order 2 in general case. The group $G$ will be presented as $G=\langle t| \emptyset>$ for oriented case while $G=<t \mid t^{2}=1>$ for general case. Let $\alpha: F=F\left(a_{1}, \ldots, a_{n}, b_{1}, \ldots, b_{n}\right) \rightarrow G$ be a homomorphism defined by $\alpha\left(a_{i}\right)=1, \alpha\left(b_{i}\right)=t$ for $i=1, \ldots, n$. We denote ${ }^{-}: \mathbf{Z} G \rightarrow \mathbf{Z} G$ an involution defined by $\overline{\left(\Sigma n_{i} t^{i}\right)}=\Sigma n_{i} t^{-i}$. Hereafter we work on orientation preserving equivalence and simply call it equivalence. But it will be easy to modify statements to adapt to non orientation preserving case.

Next we define Jacobian matrix $J \varphi_{*}$ which is associated with a plat $\varphi$

$$
J \varphi_{*}=\left(\begin{array}{ll}
\left(\frac{\partial \varphi\left(a_{\imath}\right)}{\partial a_{\jmath}}\right)_{i, J=1, \ldots, n} & \left(\frac{\partial \varphi\left(a_{i}\right)}{\partial b_{3}}\right)_{i, J=1, \ldots, n} \\
\left(\frac{\partial \varphi\left(b_{2}\right)}{\partial a_{\jmath}}\right)_{\imath, J=1, \ldots, n} & \left(\frac{\partial \varphi\left(b_{i}\right)}{\partial b_{j}}\right)_{\imath, J=1, \ldots, n}
\end{array}\right)^{\alpha}
$$

Here a matrix $\left(c_{i j}\right)^{\alpha}$ is denoted by $\left(\alpha\left(c_{i j}\right)\right)$. The symbol $\frac{\partial}{\partial x}$ denotes free derivative of Fox [5]. We denote four submatrices of $J \varphi_{*}$ by $A, B, C$ and $D$ as follows:

$$
J \varphi_{*}=\left(\begin{array}{cc}
C & A \\
D & B
\end{array}\right)
$$

where the four matrices are size of $n \times n$. The submatrix $\left(\begin{array}{l}A \\ B\end{array}\right)$ of $J \varphi_{*}$ is important for our purpose. We denote it by $R \varphi_{*}$. Namely $R \varphi_{*}=\left(\begin{array}{l}A \\ B\end{array}\right)$.

\section{The main results}

THEOREM 1. Suppose that two plats $[\varphi],[\psi] \in B_{2 n}$ are equivalent. Let $R \varphi_{*}=\left(\begin{array}{l}A \\ B\end{array}\right)$ and $R \psi_{*}=\left(\begin{array}{c}A^{\prime} \\ B^{\prime}\end{array}\right)$. Then there are $n \times n$ unımodular matrices $U, G$ and an $n \times n$ matrix $W$ which satısfy 


$$
\left(\begin{array}{cc}
U & 0 \\
W & { }^{*} U^{-1}
\end{array}\right)\left(\begin{array}{l}
A \\
B
\end{array}\right) G=\left(\begin{array}{c}
A^{\prime} \\
B^{\prime}
\end{array}\right)
$$

where, for $U=\left(u_{i j}\right),{ }^{*} U$ denotes ${ }^{t} \bar{U}$, that is ${ }^{*} U=\left(\overline{u_{i j}}\right)$.

To prove the theorem the following is a key.

LEMMA 2. If $\varphi$ belongs to $K_{2 n}$, then $J \varphi_{*}$ has a form $\left(\begin{array}{cc}U & 0 \\ W & { }^{*} U^{-1}\end{array}\right)$ where $U$ and $W$ are as in Theorem 1.

We consider equivalence relation between matrices like $R \varphi_{*}$.

Definition 5. Two matrices $R \varphi_{*}=\left(\begin{array}{l}A \\ B\end{array}\right)$ and $R \psi_{*}=\left(\begin{array}{l}A^{\prime} \\ B^{\prime}\end{array}\right)$ are said equivalent if and only if there are $n \times n$ unimodular matrices $U, G$ and an $n \times n$ matrix $W$ which satisfy

$$
\left(\begin{array}{cc}
U & 0 \\
W & { }^{*} U^{-1}
\end{array}\right)\left(\begin{array}{l}
A \\
B
\end{array}\right) G=\left(\begin{array}{c}
A^{\prime} \\
B^{\prime}
\end{array}\right) .
$$

In term of this equivalence we can restate Theorem 1 as two plats $\varphi$ and $\psi$ are equivalent only if $R \varphi_{*}$ and $R \psi_{*}$ are equivalent. We choose a nice representative from an equivalence class of $R \varphi_{*}$.

TheOREM 2. Let $\varphi$ be a plat and let $R \varphi_{*}=\left(\begin{array}{l}A \\ B\end{array}\right)$. Then $\left(\begin{array}{l}A \\ B\end{array}\right)$ is equivalent to a matrix whıch has a form

$$
\left(\begin{array}{cc}
0 & 0 \\
0 & A_{22} \\
1 & * \\
0 & B_{22}
\end{array}\right)
$$

where $A_{22}$ and $B_{22}$ are $(n-1) \times(n-1)$ matrices while $A$ and $B$ are $n \times n$ matrices.

We call a matrix which has the form as in Theorem 2 a reduced form of $R \varphi_{*}$.

THEOREM 3. Let $\varphi$ and $\psi$ be plat representation of knots. Suppose that $\varphi$ and $\psi$ are equivalent. Let

$$
R \varphi_{*} \sim\left(\begin{array}{cc}
0 & 0 \\
0 & A_{22} \\
1 & * \\
0 & B_{22}
\end{array}\right), \quad R \psi_{*} \sim\left(\begin{array}{cc}
0 & 0 \\
0 & A_{22}^{\prime} \\
1 & * \\
0 & B_{22}^{\prime}
\end{array}\right)
$$

Then there are unimodular matrices $U$ and $G$ and a matrix $W$ which satısfy

$$
\begin{gathered}
A_{22}^{\prime}=U A_{22} G \\
B_{22}^{\prime}=W A G+{ }^{*} U^{-1} B_{22} G .
\end{gathered}
$$


Remark 1. The first equation (i) of Theorem 3 shows $A^{\prime}=U A G^{-1}$. This means the matrices $A$ and $A^{\prime}$ are similar. Let us substitute $G$ in the second equation (ii) of Theorem 3 by $A^{\prime-1} U A$. Then we have

$$
{ }^{*} W+B A^{-1}={ }^{*} U B^{\prime} A^{\prime-1} U .
$$

The entries of the matrix $B A^{-1}$ are elements of the quotient field, say $F$, of $\mathbf{Z} G$. Then $B A^{-1}$ can be regarded as a bilinear form from $\mathbf{Z} G \times \mathbf{Z} G \rightarrow F / \mathbf{Z} G$. So is $B^{\prime} A^{\prime-1}$. The equation (*) means the bilinear form $B A^{-1}: \mathbf{Z} G \times \mathbf{Z} G \rightarrow F / \mathbf{Z} G$ and $B^{\prime} A^{\prime-1}$ : $\mathbf{Z} G \times \mathbf{Z} G \rightarrow F / \mathbf{Z} G$ are isomorphic. Hence we proved that an isomorphism class of $B A^{-1}$ is an invariant of the equivalence class of $\left(\begin{array}{l}A \\ B\end{array}\right)$.

If we substitute $t$ by -1 then Theorem 3 holds for non orientable case.

CoRollary. For a matrix $\left(\begin{array}{c}A \\ B\end{array}\right), g c d_{i, j=1, \ldots, n}\left(a_{i j}\right)$ and $\operatorname{det} B \bmod g c d_{i, j=1, \ldots, n}\left(a_{i \jmath}\right)$ are, up to multiplication of units, invariants of an equivalence class of $\left(\begin{array}{l}A \\ B\end{array}\right)$.

Remark 2. Corollary above holds for non orientation preserving case if we substitute $t$ by -1 .

\section{Examples}
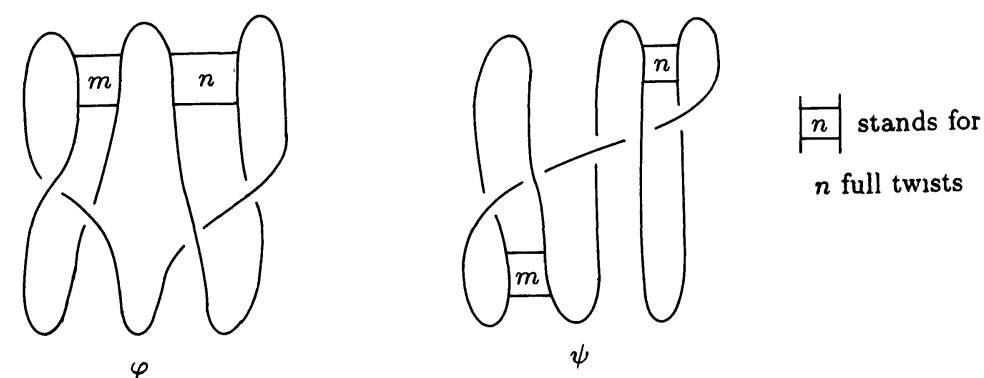

$n$ full twists

Figure 6

Let us consider the following two plats $\varphi$ and $\psi$ (Figure 6).

Here we denote $\operatorname{gcd}(4 m-1,4 n-1)$ by $d$ and suppose that $d$ is neither 1 nor 3 . To simplify calculation we substitute $t$ with -1 . Then

$$
\left(R \varphi_{*}\right)^{t=-1}=\left(\begin{array}{c}
A \\
B
\end{array}\right)^{t=-1} \sim\left(\begin{array}{ccc}
0 & 0 & 0 \\
0 & 1-4 m & 0 \\
0 & 0 & 1-4 n \\
1 & * & * \\
0 & 1-2 m & 0 \\
0 & -1+4 m & 1+4 n
\end{array}\right)
$$


and

$$
\left(R \psi_{*}\right)^{t=-1}=\left(\begin{array}{l}
A^{\prime} \\
B^{\prime}
\end{array}\right)^{t=-1} \sim\left(\begin{array}{ccc}
0 & 0 & 0 \\
0 & 1-4 m & 0 \\
0 & 0 & 1-4 n \\
1 & * & * \\
0 & 1+4 m & 0 \\
0 & -1+4 m & 1+4 n
\end{array}\right) .
$$

We have $\operatorname{det} B_{22}^{\prime}-\operatorname{det} B_{22}=(1+4 n)(1+4 m-1+2 m)=6 m(4 n-1+2) \equiv 3 \bmod d$ and $\operatorname{gcd}_{i, j=1, \ldots, n}\left(a_{i j}\right)=d$. Since $d$ is neither 1 or $3,3 \not \equiv 0 \bmod d$. Thus $\operatorname{det} B_{22} \not \equiv \operatorname{det} B_{22}^{\prime} \bmod$ $\operatorname{gcd}_{\imath, j=1, \ldots, n}\left(a_{i j}\right)$. This implies that $\left(\begin{array}{l}A \\ B\end{array}\right)$ and $\left(\begin{array}{l}A^{\prime} \\ B^{\prime}\end{array}\right)$ are not equivalent. In conclusion we can say that $\varphi$ and $\psi$ are not equivalent by Theorem 1 . Note that these two plats are equivalent as knots.

\title{
RefERENCE
}

[1] J.S. Birman and H.M. Hilden, On the mapping class groups of closed surfaces as covering spaces, Ann. Math. Studies 66 (1971), 81-115.

[2] J.S. Birman and H.M. Hilden, Heegaard splittings of branched coverings of $S^{3}$, Trans. Amer. Math. Soc. 213 (1975), 315-352.

[ 3 ] J.S. Birman, On the equivalence of Heegaard splittings of closed, orientable 3-manifolds, Ann. Math. Studies 84 (1975), 137-164.

[4] J.S. Birman, On the stable equivalence of plat representations of knots and links, Canad. J. Math. 28 (1976), 264-290.

[ 5 ] R.H. Fox, Free differential calculus I, Ann. of Math. 57 (1953), 547-560.

[6] S. Fukuhara and J. Kanno, Extended Alexander matrices of 3-manifolds I, Tokyo J. Math. 8 (1985), 107-120.

[ 7 ] S. Fukuhara, Invariants of equivalence classes of plats, J. Fac. Sci. Univ. Tokyo, Sect. IA, Math. 40 (1993), 503-516.

[8] H.M. Hilden, Generators for two groups related to the braid group, Pacific J. Math. 59 (1975), 475-486.

[9] M. Lustig and Y. Moriah, Nielsen equivalence in Fuchsian groups and Seifert fibered spaces, Topology 30 (1991), 191-204.

[10] M. Lustig and Y. Moriah, Generalized Montesinos knots, tunnels and N-torsion, Math. Ann. 295 (1993), 167-189.

[11] J.M. Montesinos, Minimal plat representations of prime knots and links are not unique, Canad. J. Math. 28 (1976), 161-167.

[12] J.M. Montesinos and C. Safont, On the Birman invariants of Heegaard splittings, Pacific J. Math. 132 (1988), 113-142.

\author{
TSUDA COLLEGE \\ TSUDA-MACHI, KODAIRA-SHI \\ TOKYO 187 , JAPAN
}

